\title{
Les habitants au cœur de la décision
}

Conférence de consensus - Lille 25, 26, 27 juin 2003

\section{Centre Ressource du Développement Durable (CERDD)}

\section{OpenEdition \\ 12 Journals}

\section{Édition électronique}

URL : http://journals.openedition.org/developpementdurable/997

DOI : 10.4000/developpementdurable.997

ISSN : 1772-9971

Éditeur

Association DD\&T

\section{Référence électronique}

Centre Ressource du Développement Durable (CERDD), « Les habitants au cœur de la décision», Développement durable et territoires [En ligne], Points de vue (2003-2010), mis en ligne le 09 octobre 2003, consulté le 20 avril 2019. URL : http://journals.openedition.org/developpementdurable/997 ; DOI : 10.4000/developpementdurable.997

\section{Ce document a été généré automatiquement le 20 avril 2019.}

Développement Durable et Territoires est mis à disposition selon les termes de la licence Creative Commons Attribution - Pas d'Utilisation Commerciale 4.0 International. 


\section{Les habitants au cœur de la décision}

Conférence de consensus - Lille 25, 26, 27 juin 2003

Centre Ressource du Développement Durable (CERDD)

\section{NOTE DE L'ÉDITEUR}

Les deux textes qui suivent (les conclusions de cette conférence de consensus et la version « Autrement dit » de ces conclusions), ont été rédigés par les conférenciers. Ils sont également accessibles sur le site des Assises Nationales du Développement Durable de Lille en juin 2003.

1 Le Conseil Régional Nord-Pas-de-Calais a pris l'initiative de réunir une conférence de consensus. A la différence d'une conférence de citoyens, la conférence réunit un ensemble de personnes pour confronter sur un même sujet des expériences différentes afin d'accoucher de propositions communes. Le Conseil régional Nord Pas-de-Calais puis la Région qui accueillera les prochaines Assises pourraient se relayer pour assurer la continuité de ce travail avec l'ensemble des co-organisateurs.

2 Le terme de « consensus " renvoie à un socle commun de propositions pour construire une bonne participation. En aucun cas il ne sous-estime l'importance des différences, autrement dit le dissensus, qui permet de poser de bonnes questions, de faire entendre de vraies revendications.

C'est pourquoi les participants mettent en interpellation à la fois ce qui fait accord et ce qui fait encore questionnement et ouvre un nouvel espace de débat.

3 Nous, participants, présents à la conférence de consensus de Lille, déclarons que les travaux ont conduit à des conclusions qui constituent la première étape d'une réflexion qui s'engage.

\section{Le socle commun}

1. La qualité de l'information est la base de la participation. 
L'habitant doit pouvoir avoir accès à une information donnée au bon moment, complète et contradictoire, compréhensible par le plus grand nombre.

L'information doit respecter 4 principes :

- Un principe de transparence : tout doit être dit sauf ce qui est interdit par la loi. La confidentialité des informations doit rester l'exception, et en tout état de cause être expliquée.

- Un principe d'égalité de moyens. Les habitants, les élus, les techniciens, les acteurs économiques... tous les acteurs doivent pouvoir produire leur propre information. L'information n'est pas seulement donnée par le maître d'ouvrage : elle doit être plurielle et contradictoire.

- Un principe d'adaptation : les supports d'information doivent s'ajuster à la culture des lieux et des gens. Les projets doivent aussi se discuter sur les lieux où vivent les gens.

- Un principe de réciprocité : l'échange d'information dans un débat suppose des capacités d'écoute de chacun et du temps pour entendre et comprendre.

\section{Il est essentiel qu'il y ait une égalité d'accès à la prise de parole}

- Toute formation à la prise à la parole suppose parallèlement un apprentissage de l'écoute.

- Toute demande d'explication est légitime. Au cours d'un débat, on doit pouvoir aussi se faire expliquer ce que l'on n'a pas compris.

- Toute parole qui s'échange dans les débats doit être enregistrée, retranscrite et diffusée.

- Tout ne passe pas forcément par l'écrit et la parole : il faut diversifier les modes d'expression (la parole, l'écrit, le dessin, le théâtre, la promenade...).

8 3. Il faut garantir une égalité d'accès des citoyens, y compris les plus démunis, à la discussion démocratique.

- Plus on diversifie les formes de la participation et plus on se donne de chances de mobiliser les gens.

- Tout dispositif de participation doit pouvoir prendre en compte l'opinion et les intérêts des populations exclues qu'elles soient ou non présentes physiquement.

- L'efficacité des dispositifs de participation repose très largement sur l'importance des moyens matériels qu'on leur consacre.

- Il y aura d'autant plus de monde dans les dispositifs de participation que ceux-ci auront une influence réelle sur la décision.

- La participation des associations est nécessaire mais pas suffisante pour que les opinions des citoyens soient représentées.

- Le tirage au sort constitue l'un des modes de représentation possible des citoyens ordinaires et non concernés.

- Il faut légiférer pour donner des moyens d'agir aux citoyens membres ou animateurs des instances participatives (crédit d'heures, formation, ...).

\section{La participation nécessite d'afficher un cadre et des règles du jeu}

- Il est souhaitable que les règles du jeu soient co-élaborées et acceptées par les différentes parties.

- Les règles du jeu doivent faire l'objet d'un bilan régulier pour être ajustées chemin faisant.

- Il faut encourager les chartes locales et intercommunales de participation et faire vivre celles qui existent.

- La loi n'est pas suffisante mais en imposant un minimum, elle est un levier pour enclencher la participation. 

habitants.

- L'expertise des habitants ne se limite pas à son cadre de vie : elle doit pouvoir s'exercer du local au global. Il faut de la même façon reconnaître l'expertise technique de certains habitants et se donner les moyens de la mobiliser.

- Pour tout projet, le maître d'ouvrage doit mettre à disposition un budget permettant aux habitants de recourir à la contre-expertise.

- Les techniciens des collectivités doivent pouvoir jouer un rôle d'expert auprès des habitants. Il faut pour cela adapter leur mission.

1 6. La participation doit prendre en compte le temps et les échelles du développement durable

- La participation doit pouvoir suivre son propre rythme qui n'est pas forcément celui des mandats électoraux, ni celui de l'administration.

- L'emboîtement des échelles de territoire nécessite de travailler en réseau. Il faut donner des moyens aux acteurs de la participation pour leur permettre de passer à des échelles géographiques supérieures.

- Les temps du débat, de la discussion et de la décision doivent être aménagés d'une manière compatible avec les modes de vie des citoyens appelés à y participer.

- Le calendrier du projet doit être clarifié en permanence.

7. La participation ouvre un espace de délibération dans le processus de décision des politiques publiques.

- Chacun (élu ou société civile) a droit à mettre des sujets à l'ordre du jour.

- Le problème doit être clairement posé avant toute solution, avant toute mise en œuvre ou arbitrage.

- La qualité du débat résulte d'une méthode conçue comme la construction d'accords et de désaccords.

- Un bilan de la participation précède l'arbitrage : il valorise les apports créatifs et inflexions des habitants. Il mentionne les justifications des élus sur les points non retenus.

- L'élu veille à la reconnaissance ou à l'ouverture d'espaces démocratiques de coproduction des choix. Ses votes, son action sur la mise en œuvre, veillent à garantir l'intérêt général par la prise en compte alternative de la participation préalable.

\section{Il n'y a pas de participation sans évaluation.}

- L'évaluation démocratique implique forcément les acteurs engagés dans le processus.

- Deux types d'évaluation sont à croiser :

- L'évaluation de la qualité du processus de participation lui-même de façon à rechercher des améliorations pour la suite.

- L'évaluation collective de l'effet de la participation sur la décision

- L'évaluation participative est enrichie par des apports multiples: des échanges d'expériences, des diagnostics partagés, des audits extérieurs, des observatoires de la participation...Elle doit conduire à se poser des questions pour enrichir l'action.

- Il est nécessaire de veiller, dès le départ d'un processus de participation, à s'organiser pour constituer la mémoire qui permettra ensuite une évaluation.

- L'évaluation est en elle-même un processus de participation : elle enrichit, développe des compétences, donne la capacité de participer. 


\section{Les questionnements mis en débat}

Deux questions ont fait principalement débat au cours de cette conférence

- La question de la représentativité

Comment les démarches participatives tirent leur légitimité : Est-ce de la représentation sociologique? Est-ce dans leur capacité à mobiliser le plus grand nombre? Est-ce dans l'expression de la diversité des arguments et des usages? Existe-t-il encore des formes de légitimité qui doivent être prises en compte et lesquelles?

- La question du partage de la décision : Une démocratie participative peut-elle se concevoir sans co-décision? Autrement dit, l'objectif central de la participation est-il de co-produire de la décision ou simplement de fabriquer de bons citoyens?

\section{Les suites de la conférence de consensus}

Les participants de la Conférence de consensus s'engagent dans leurs organismes :

- à faire débattre des conclusions proposées dans leur instance.

- à indiquer les conclusions qu'elles en tirent pour elles-mêmes.

- à rendre publique ses conclusions.

Ils souhaitent que les co-financeurs des Assises du développement durable de Lille en fassent de même.

17 Les participants de la Conférence de Consensus souhaitent que les travaux qu'ils ont lancés sur les principes de la participation soient poursuivis par un travail continu sur des recommandations pratiques.

\section{La participation et le développement durable. Bilan de la conférence de consensus, "AUTREMENT DIT »}

Le développement durable c'est s'occuper du social, de l'environnement, de l'économie. Comme tout le monde n'a pas les mêmes intérêts, il faut discuter. Pour que les habitants discutent le mieux possible, 49 personnes ont réfléchi aux bonnes conditions de la participation pendant les Assises du Développement Durable. Voilà ce qu'elles ont conclu :

19 1) Chacun a le droit de savoir

- Rien ne doit être caché. Si on interdit de montrer quelque chose il faut dire pourquoi.

- Les informations ne se limitent pas à des documents illisibles produits par les services des élus.

- Les autres façons de faire circuler l'information doivent être prises en compte et aidées.

- Chacun doit parler. Chacun doit écouter l'autre. Il faut laisser le temps de comprendre.

- Les projets se discutent aussi où vivent les gens.

\section{2) Chacun a le droit de donner ses idées et son avis}

- Discuter c'est savoir parler et aussi savoir écouter.

- Pour comprendre, une bonne explication est indispensable, et un travail de préparation en petit groupe.

- En venant, on sait ce que deviendra ce que l'on dit. 
- Le débat est écrit. Chacun peut venir le lire. Au moment de la décision, il est montré tout ce qui a été pris en compte. Il faut dire pourquoi certaines choses ont été refusées.

- Leaders et associations doivent être reçus, aidés et entendus.

- Il n'y a pas que la discussion qui fait avancer les choses. Venir sur le terrain, faire un spectacle, vivre ensemble compte aussi.

21 3) Les plus pauvres ne viennent souvent pas au débat. Ce n'est pas une raison pour ne pas prendre en compte leurs besoins

- Celui qui s’informe, qui organise le débat est indépendant des élus.

- Les associations, c'est important mais cela ne suffit pas.

- Participer à un débat demande des salles, du papier, des copies, des déplacements. La loi doit prévoir les moyens.

- En plus de ceux qui viennent au débat, on peut ajouter des gens tirés au sort.

- Il n'y a pas que dans les débats qu'on discute. Sur le terrain, cela fait parfois plus de monde.

- Les plus pauvres méritent une écoute attentive. La réponse à leurs besoins est indispensable.

- Sans moyens, la participation est mauvaise.

- On ne viendra pas si ça sert à rien.

\section{4) Il n'y a pas que l'avis des experts ! Il y a aussi l'avis des habitants}

- Ce sont quand même ceux qui vivent là qui ont leur mot à dire.

- Et ce qu'ils disent n'est pas plus bête qu'autre chose, aussi bien sur leur quartier, que sur l'état de la planète.

- Des moyens devraient aider les habitants à mieux présenter ce qu'ils veulent.

- Les techniciens des élus devraient même pouvoir les aider.

5) Participer c'est se mêler des projets petits ou grands, des projets pour tout de suite ou pour le futur : c'est du développement durable

- Pendant qu'on débat, il ne faut pas qu'ils décident ailleurs ou sans attendre notre travail.

- Chacun réfléchit dans son coin. Faire un réseau ensemble rend plus efficace.

- Il n'y a pas que les moments d'élection qui comptent.

- Si quelque chose se décide, les habitants doivent être actifs avant, pendant et après.

- Le calendrier doit être affiché.

- Il ne faut pas balader les gens avec des procédures administratives.

6) Ce n'est pas la peine de faire travailler les habitants si des décisions sont déjà prises, même en partie

- Les habitants ont aussi le droit de choisir de quoi on parle.

- Avant de débattre sur un projet, il faut débattre sur : quel problème veut-on résoudre?

- Si les habitants ont d'autres idées de solutions, il faut les aider à préparer l'autre scénario, leur donner des moyens.

- On doit faire la liste des accords et des désaccords pour avancer.

- Chaque travail des habitants sur un projet doit être suivi :

- d'un relevé de tout ce qu'ils ont apporté.

- de tout ce qu'il leur a été refusé, avec la raison pour laquelle cela leur a été refusé.

- L'élu doit reconnaître ceux qui réfléchissent sur le terrain. Il doit faciliter leur travail, ou la naissance de tels groupes.

- Avec toutes ces propositions, il peut construire l'intérêt général.

\section{7) Pas de participation sans évaluation}

- Participer, c'est jouer son rôle de citoyen, c'est rendre les projets meilleurs.

- On décide ensemble comment on évalue. 
- Il faut regarder et évaluer :

- Comment s'est faite la participation elle-même.

- Comment ce qui a été dit a été suivi d'effet.

- Evaluer ensemble, c'est aussi raconter ses expériences, voir ce qu'en disent les autres, regarder ce qui c'est déjà fait. Tout cela fait progresser.

- C'est un animateur qui accompagne l'évaluation.

- Dès le départ, on fait attention à conserver les comptes rendus.

- En évaluant, on dynamise la participation.

\section{8) Il faut dès le départ être d'accord sur la méthode}

- Ceux qui proposent le débat proposent aussi une méthode. Il faut un accord.

- Au fur et à mesure, on voit si ça marche.

- Chacun respecte les règles une fois qu'on les a acceptées.

- C'est mieux quand les communes ou intercommunalités ont fait une charte de participation.

- La loi doit obliger à un minimum.

9) La participation, ce n'est pas demander l'avis des habitants quand tout est fini. Participer, c'est « faire avec ».

\section{ANNEXES}

Liste nominative des conférenciers

Eric Andrieu (UNADEL), Catherine Atger-Lhotellerie (CERTU), Magali Bardou (MEDD), Thierry Benoît (FARRE), Emmanuel Bertin (GEANTS), Bernard Birsinger (ville de Bobigny), Catherine Bertram (Mission bassin minier), Maurice Blanc (université Nancy 2), Marie Blandin (Sénat), Loïc Blondiaux (IEP Lille), Céline Braillon (CNV), Valérie Caron (ville de Loos-en-Gohelle), Claude Chardonnet (cabinet C\&S), Jean-Pierre Chaussade (EDF), Claude Coquelle (COPAS), Jean Culdaut (CNCE), Abdelkader Damani (Robins des Villes), Jérome Dancoisne (EDA/MNE), Richard Dartout (Agence de l'eau Seine Normandie), Pierre Davenne (ville de Linselles), Christine Degrugillier (ville de Loos-en-Gohelle), Serge Depaquit (ADELS), Michaël Dereux (Ecomaires), Gabriel Dewalle (Confédération Paysanne), Alain Dubois (DIREN), Jean Marc Dziedzicki (RFF), Jamal Ejjalty (ville de Lille), Jean Michel Fourniau (INRETS), Stéphane Gornikowski (CFDT), Jean Marie Gueuret (AGUR), Edmond Henguelle (Conseil de développement-Pays des 7 vallées), Michel Joncquel (Regards d'habitants), Maud Lelièvre (ville de Saint Denis), Anne Le Guillou (ARENES), Gérard Léval (université Lille III), Catherine Marette (CNCE), Philippe Marzolf (CNDP), Michel Mousel (4D), Magali Nonjon (CRAPS), Pascal Percq (ville de Lille), René Perier (DDE), Nathalie Pinelli (SIRVAL), Isabelle Pisani (IPA Consultants), Albert Prissette (DAL), Jacques Remond (CARNACQ), Colette Lassalas-Ronxin (ville d'Amiens), Yves Sintomer (université Paris 8/ Berlin), Laurence Thiery (Conseil Régional du Nord-Pas-deCalais), Philippe Vaesken (E2I). 


\section{RÉSUMÉS}

Le développement durable se construit sur la confrontation des intérêts sociaux, environnementaux, économiques. Sans démocratie, cette confrontation ne trouve pas de lieu de débat et d'arbitrage. La conférence de consensus, réunie pendant ces Assises du développement durable a fait travailler 49 personnes ayant une pratique de la participation: associatifs, syndicalistes, techniciens, experts, universitaires, élus... sur le thème «les habitants au cœur de la décision : les conditions de la participation ». L'objectif était de creuser les conditions de la participation des gens et de s'interroger sur la possible co-production de la décision publique.

\section{INDEX}

Mots-clés : participation, démocratie participative, délibération, conférence de consensus, recommandations méthodologiques 